University of Wollongong

Research Online

Australian Institute for Innovative Materials -

Papers

Australian Institute for Innovative Materials

$1-1-2018$

\title{
Unraveling the effect of salt chemistry on long-durability high-phosphorus- concentration anode for potassium ion batteries
}

\author{
Wenchao Zhang \\ University of Wollongong,wz990@uowmail.edu.au \\ Zhibin Wu \\ University of Wollongong, zw120@uowmail.edu.au \\ Jian Zhang \\ Changsha University of Science And Technology \\ Guoping Liu \\ Guangdong University of Technology \\ Nai-Hsuan Yang \\ National Taiwan University
}

See next page for additional authors

Follow this and additional works at: https://ro.uow.edu.au/aiimpapers

Part of the Engineering Commons, and the Physical Sciences and Mathematics Commons

Research Online is the open access institutional repository for the University of Wollongong. For further information contact the UOW Library: research-pubs@uow.edu.au 


\title{
Unraveling the effect of salt chemistry on long-durability high-phosphorus- concentration anode for potassium ion batteries
}

\author{
Abstract \\ Phosphorus-based anode materials are of considerable interest for grid-scale energy storage systems \\ due to their high theoretical capacity. Nevertheless, the low electrical conductivity of $P$, large volume \\ changes during cycling, and highly-reactive phosphide surface are hindering their potential applications. \\ Herein, outstanding long-term cycling stability with high retained potassium storage capacity (213.7 mA h \\ g-1over 2000 cycles) was achieved via the introduction of an alternative potassium \\ bis(fluorosulfonyl)imide (KFSI) salt and by using a layered compound (GeP5) with a high phosphorus \\ concentration as anode material. Fourier transform infrared spectroscopic mapping results suggest that \\ KFSI salt helps to form an uniform solid electrolyte interphase (SEI) layer and reduces the side reactions \\ at the electrode/electrolyte interface, thus enhancing the cycling performance. In-operando synchrotron \\ $\mathrm{X}$-ray diffraction analysis has revealed the synergistic reaction mechanisms of the K-P and K-Ge reactions. \\ These findings indicate the enormous potential of phosphorus-based anodes for high-performance \\ potassium ion batteries and can attract broad interest for regulating the SEI layer formation through \\ manipulating the salt chemistry. \\ Disciplines \\ Engineering | Physical Sciences and Mathematics

\section{Publication Details} \\ Zhang, W., Wu, Z., Zhang, J., Liu, G., Yang, N., Liu, R., Pang, W., Li, W. \& Guo, Z. (2018). Unraveling the effect \\ of salt chemistry on long-durability high-phosphorus-concentration anode for potassium ion batteries. \\ Nano Energy, 53 967-974.

\section{Authors} \\ Wenchao Zhang, Zhibin Wu, Jian Zhang, Guoping Liu, Nai-Hsuan Yang, Ru-Shi Liu, Wei Kong Pang, Wenwu \\ $\mathrm{Li}$, and Zaiping Guo
}




\section{Unraveling the effect of salt chemistry on long-}

\section{durability high-phosphorus-concentration anode for}

\section{potassium ion batteries}

Wenchao Zhang ${ }^{1}$, Zhibin $\mathrm{Wu}^{1}$, Jian Zhang ${ }^{3}$, Guoping Liu ${ }^{2}$, Nai-Hsuan Yang ${ }^{4}$, Ru-Shi Liu ${ }^{4}$, Wei

Kong Pang ${ }^{1 *}$, Wenwu $\mathrm{Li}^{2 *}$, Zaiping Guo ${ }^{1 *}$

[1] Institute for Superconducting \& Electronic Materials, School of Mechanical, Materials, Mechatronics \& Biomedical Engineering, Faculty of Engineering and Information Sciences, University of Wollongong, NSW 2500, Australia.

[2] School of Materials and Energy, Guangdong University of Technology, No. 100 Waihuan Xi

Road, Guangzhou Higher Education Mega Center, Guangzhou 510006, P. R. China.

[3] College of Automotive and Mechanical Engineering, Changsha University of Science and Technology, Changsha 410114, China

[4] Department of Chemistry, National Taiwan University, Taipei 106, Taiwan

*Corresponding author. Email: wkpang@uow.edu.au (W.P.); wenwuli@hust.edu.cn (W.L.); zguo@uow.edu.au (Z.G.) 


\title{
KEYWORDS
}

potassium ion batteries; salt chemistry; phosphorus-based anode; long-durability; synergistic reaction

\begin{abstract}
Phosphorus-based anode materials are of considerable interest for grid-scale energy storage systems due to their high theoretical capacity. Nevertheless, the low electrical conductivity of P, large volume changes during cycling, and highly-reactive phosphide surface are hindering their potential applications. Herein, outstanding long-term cycling stability with high retained potassium storage capacity (213.7 $\mathrm{mA} \mathrm{h} \mathrm{g}^{-1}$ over 2000 cycles) was achieved via the introduction of an alternative potassium bis(fluorosulfonyl)imide (KFSI) salt and by using a layered compound $\left(\mathrm{GeP}_{5}\right)$ with a high phosphorus concentration as anode material. Fourier transform infrared spectroscopic mapping results suggest that KFSI salt helps to form an uniform solid electrolyte interphase (SEI) layer and reduces the side reactions at the electrode/electrolyte interface, thus enhancing the cycling performance. In-operando synchrotron X-ray diffraction analysis has revealed the synergistic reaction mechanisms of the K-P and K-Ge reactions. These findings indicate the enormous potential of phosphorus-based anodes for high-performance potassium ion batteries and can attract broad interest for regulating the SEI layer formation through manipulating the salt chemistry.
\end{abstract}




\section{Introduction}

With the increased demands of electric cars and large-scale energy storage devices, highenergy density and low-cost batteries have been of great interest for the past few years ${ }^{1-7}$. While lithium ion batteries (LIBs) have dominated in portable devices and electric vehicles for decades, there is still considerable debate as to whether our limited lithium resources can meet the requirements for scalable application in grid-level storage ${ }^{8-11}$. Hence, it is necessary to broaden our research interest to consider cost-effective alternatives. Due to this concern, the other group-I elements $(\mathrm{Na}$ and $\mathrm{K})$ have attracted the focus of attention because of their similar properties to $\mathrm{Li}$ and greater abundance in the Earth's crust ${ }^{12-15}$. The standard hydrogen potential $\left(-2.71 \mathrm{~V}\right.$ vs. $\left.\mathrm{E}^{\mathrm{o}}\right)$ of $\mathrm{Na}$, however, usually translates into a lower working voltage than that of $\mathrm{Li}$ in full cells, indicating that lower energy density is achieved in sodium ion batteries (SIBs) ${ }^{16,17}$. Potassium, with abundant resources and a low standard hydrogen potential $\left(-2.93 \mathrm{~V}\right.$ vs. $\left.E^{\mathrm{o}}\right)$, close to that of lithium (-3.04 V vs. $\left.E^{\mathrm{o}}\right)$, makes potassium ion batteries (PIBs) a good candidate to replace $\operatorname{LIBs}^{18-20}$.

Carbon-based anode materials have been extensively studied for reversible K-ion storage with excellent cycling performance ${ }^{21,22}$, although their low volumetric capacity and energy density due to their low theoretical capacity are still obstacles for industrial applications ${ }^{23}$. Phosphorus as an alternative anode material has attracted numerous studies because of its high theoretical capacity $\left(2594 \mathrm{~mA} \mathrm{~h} \mathrm{~g}^{-1}\right)^{24}$. In order to overcome the drawbacks of the low electrical conductivity of phosphorus and large volume changes during cycling, alloy-based phosphides $\left(\mathrm{NiP}_{2}, \mathrm{Se}_{4} \mathrm{P}_{4}, \mathrm{Sn}_{4} \mathrm{P}_{3}, \mathrm{SnP}_{3}\right.$, etc. $\left.{ }^{25-29}\right)$ have been introduced because of the metallic feature in these compounds and their synergistic reaction mechanisms ${ }^{27,30}$. Although these phosphides have demonstrated promising cycling performance, their low $\mathrm{P}$ atomic percentages still limit their 
capacity. Recently, $\mathrm{GeP}_{5}$ has been reported as a potential anode with the highest $\mathrm{P}$ atomic percentage among all the binary phosphides. Although Ge may have high costs compared with other metal such as $\mathrm{Sn}, \mathrm{GeP}_{5}$ possess not only comparable low costs (only $16.7 \%$ atomic percentage of Ge in the compound) but also exhibits a very large specific gravimetric capacity. Combined with a density of $3.65 \mathrm{~g} \mathrm{~cm}^{-3}$, a high volumetric capacity of $6865 \mathrm{~mA} \mathrm{~h} \mathrm{~cm} \mathrm{~cm}^{-3}$ could be obtained, which would be beneficial for its application. In addition, $\mathrm{GeP}_{5}$ exhibits a layered structure similar to those of black $\mathrm{P}$ and graphite, and its electronic conductivity is 4 orders of magnitude higher than that of black $\mathrm{P}$ and similar to that of graphite, being favourable for potassium ion storage ${ }^{31}$.

Herein, we have prepared layered phosphorus-like $\mathrm{GeP}_{5}$ compound and evaluated its electrochemical performance as an anode for PIBs. Moreover, to further improve the coulombic efficiency (CE) and cycling stability, an alternative potassium salt, potassium bis(fluorosulfonyl)imide (KFSI), was employed to help the electrode form an even solid electrolyte interphase (SEI) layer and suppress the excessive side reactions. The $\mathrm{GeP}_{5}$ electrode in KFSI-ethylene carbonate/ diethyl carbonate (EC/DEC) electrolyte exhibited outstanding longterm cyclability (213.7 $\mathrm{mA} \mathrm{h} \mathrm{g}^{-1}$ over 2000 cycles) at the current density of $500 \mathrm{~mA} \mathrm{~g}^{-1}$. To the best of our knowledge, the obtained anodic cycling performance in $\mathrm{K}$ storage exceeds those of all the alloy-based anodes. The reaction mechanism of $\mathrm{GeP}_{5}$ as anode in PIBs was first investigated by in-operando synchrotron X-ray diffraction (XRD). The suggested conversion-type $\left(\mathrm{P} \rightarrow \mathrm{K}_{4} \mathrm{P}_{3}\right)$ and alloy-type ( $\mathrm{Ge} \rightarrow \mathrm{KGe}$ ) reactions synergistically buffer the volume changes during cycling, thus enhancing the integrity of the electrodes.

\section{Results and Discussion}



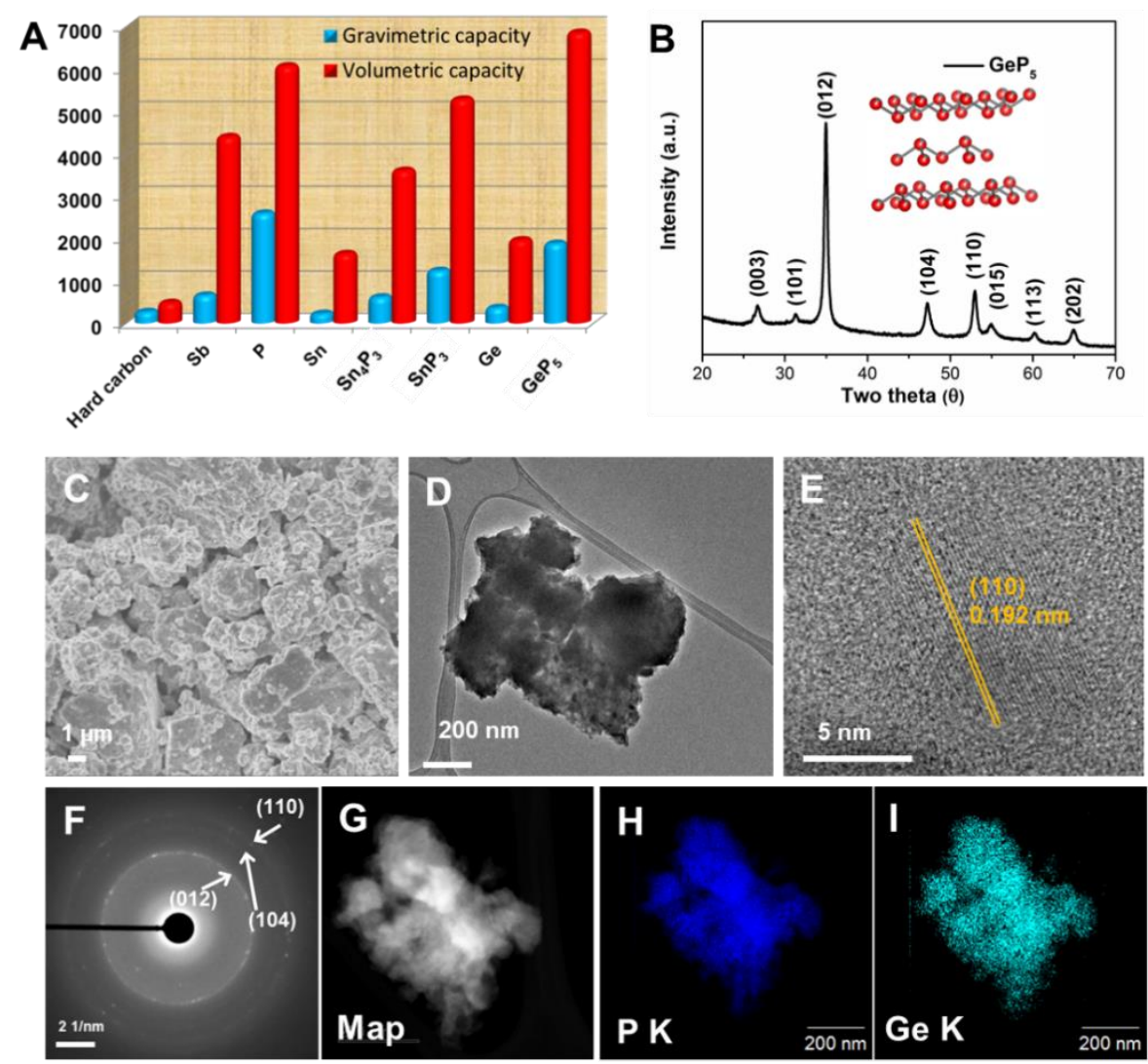

Fig. 1. Characterization of GeP5. (A) Theoretical gravimetric and volumetric capacities for various anode materials in $\mathrm{K}$-ion batteries. (B) $\mathrm{XRD}$ pattern of the $\mathrm{GeP}_{5}$ powder (with the inset showing the crystal model for $\mathrm{GeP}_{5}$ ). (C) SEM image, (D) TEM image, (E) HRTEM image, (F) selected area diffraction pattern, and (G-I) the STEM-EDS mapping of $\mathrm{GeP}_{5}$ powder.

$\mathrm{P}$ can react with $\mathrm{K}$ to form $\mathrm{K}_{3} \mathrm{P}$, leading to a high capacity $\left(2596 \mathrm{~mA} \mathrm{~h} \mathrm{~g}^{-1}\right)$, which is the highest theoretical capacity among the anodes for PIBs reported so far. The low conductivity of red phosphorus, however, and the large volume variation during cycling are blocking its application as anode material. By the introduction of Ge metal with high electrical conductivity, $\mathrm{GeP}_{5}$ with its high phosphorus concentration has a high theoretical gravimetric capacity. As 
shown in Fig. 1A, $\mathrm{GeP}_{5}$ also exhibits the highest theoretical volumetric capacity among the alloy-based anodes. The $\mathrm{GeP}_{5}$ compounds were obtained by high-energy ball-milling of Ge and red phosphorus mixtures. All of the diffraction peaks in the XRD pattern of the as-milled sample (Fig. 1B) can be assigned to $\mathrm{GeP}_{5}(R \overline{3} m$, PDF No.00-024-0455) with a layered structure (inset of Fig. 1B). No impurities were detected, implying that Ge and $\mathrm{P}$ were completely reacted and transformed to $\mathrm{GeP}_{5}$ phase. The broadened diffraction peaks indicate that the $\mathrm{GeP}_{5}$ is in nanocrystalline form. The field-emission scanning electron microscope (FESEM) image (Fig. 1C) shows the morphology, consisting of irregular agglomerated microsized particles, and the transmission electron microscope (TEM) image (Fig. 1D) presents the secondary particles, which appear as agglomerations of nanoparticles. Figure 1E shows that the spacing of the fringes is $0.283 \mathrm{~nm}$, which matches the $d_{110}$ spacing of $\mathrm{GeP}_{5}$. The corresponding selected area diffraction (SAED) pattern (Fig. 1F) can be indexed to the (012), (104), and (110) planes of GeP 5 . The scanning TEM - energy dispersive spectroscopy (STEM-EDS) elemental mapping images exhibit the uniform distributions of the Ge and $\mathrm{P}$ in the as-prepared particles, as shown in Fig. $1 \mathrm{G}-\mathrm{I}$.

\section{Electrochemical characterization and analysis of GeP5 anode}



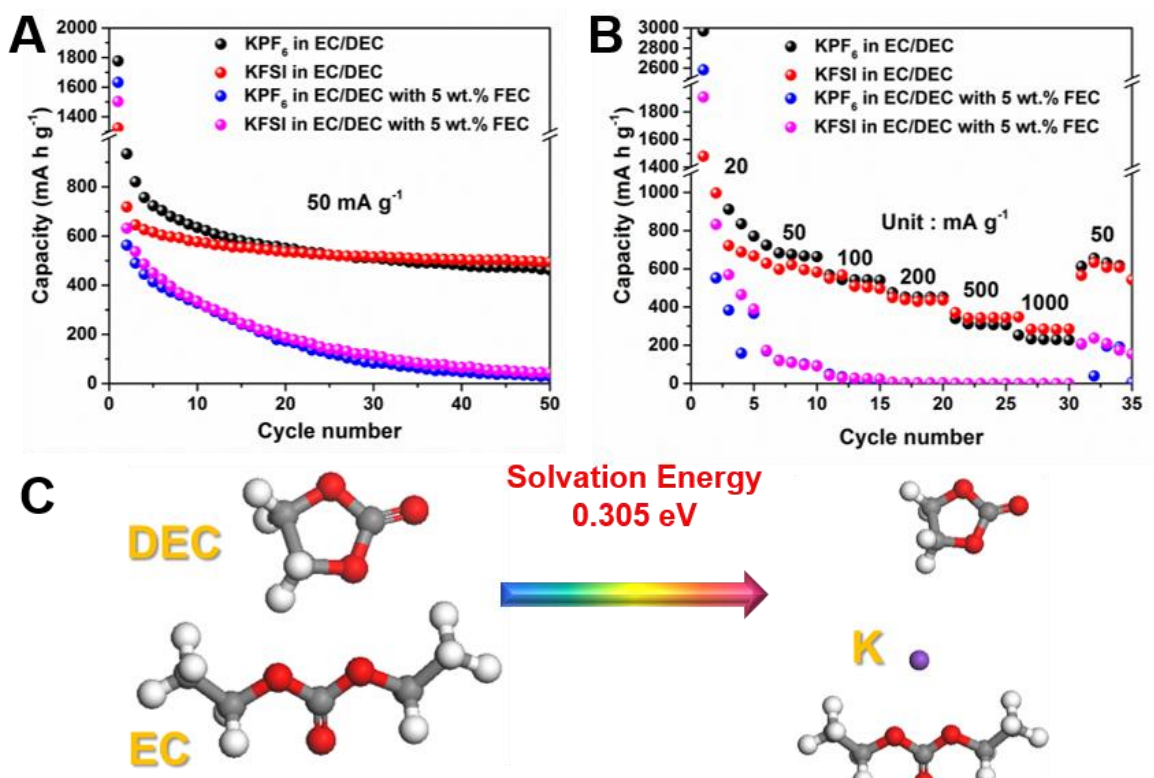

Solvation Energy

$0.305 \mathrm{eV}$
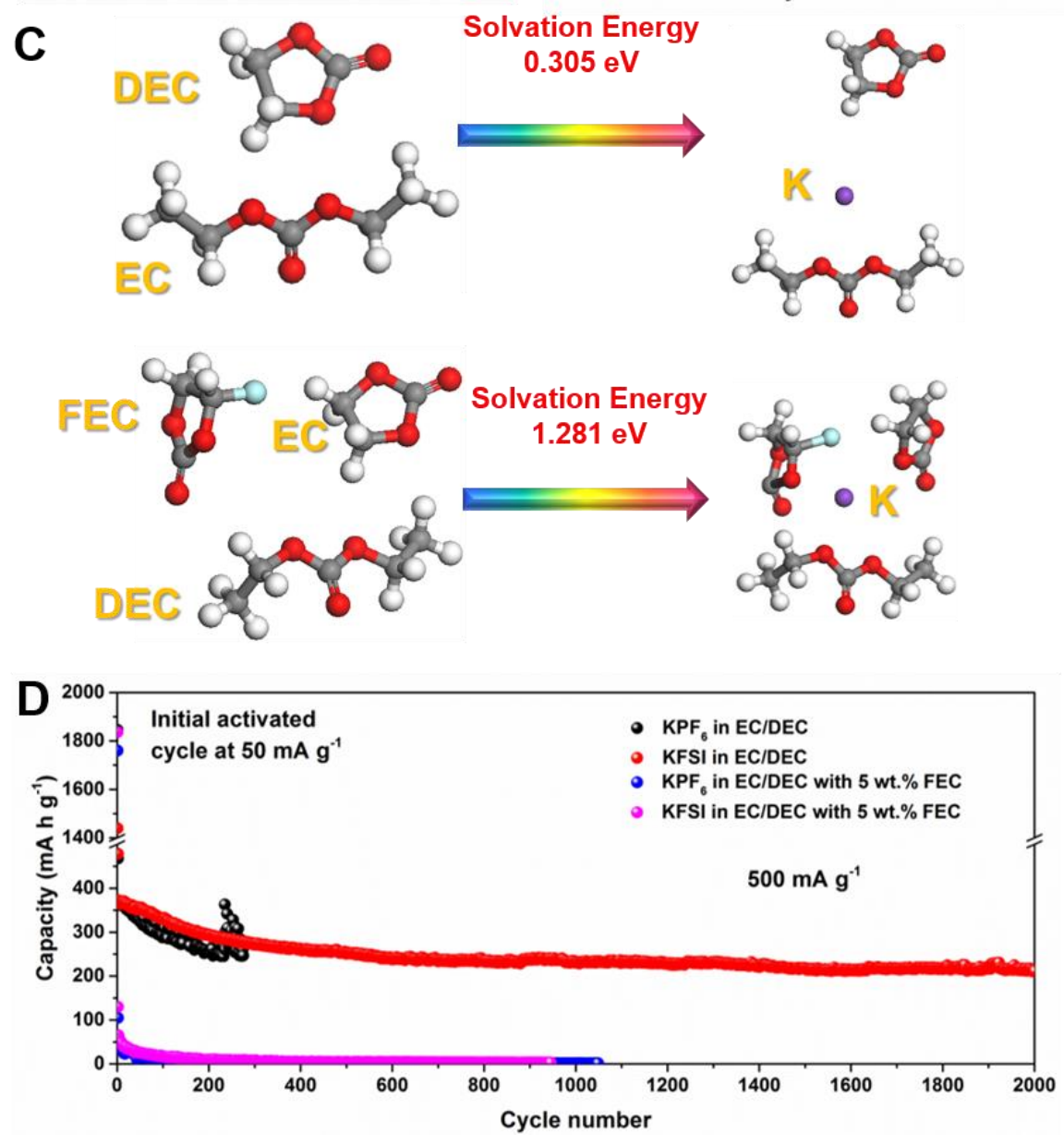

Fig. 2. Electrochemical performance of $\mathrm{GeP}_{5}$ as anode for PIBs. (A) Cycling performances of cells with various electrolytes at $50 \mathrm{~mA} \mathrm{~g}^{-1}$. (B) Rate capabilities of cells at various current densities.(C) Solvation energies estimated from the binding energy of the $\mathrm{K}^{+}(\mathrm{Y})$ clusters, where $\mathrm{Y}=\mathrm{EC}+\mathrm{DEC}$ or EC + DEC + FEC. (D) Long-term cycling performance of the cells with various electrolytes at $500 \mathrm{~mA} \mathrm{~g}^{-1}$. 
As shown in Fig. 2A, the $\mathrm{GeP}_{5}$ electrode exhibited an attractive reversible specific capacity $\left(934.5 \mathrm{~mA} \mathrm{~h} \mathrm{~g}^{-1}\right)$ at the second cycle with a slow drop afterwards in $\mathrm{KPF}_{6}$ electrolyte. The initial $\mathrm{CE}$ is only $45.81 \%$, however, which is possibly due to the high irreversible capacity caused by the SEI layer formation and the possible side reactions caused by the highly-reactive potassium metal $^{32}$. To address the problem of low initial CE and suppress the side reactions during cycling, an alternative potassium salt (KFSI) was adopted ${ }^{32,33}$. The $\mathrm{GeP}_{5}$ electrode in KFSI electrolyte presents a relatively high initial CE of $58.57 \%$ and stable cycling performance, with retained capacity of $495.1 \mathrm{~mA} \mathrm{~h} \mathrm{~g}^{-1}$ after 50 cycles at $50 \mathrm{~mA} \mathrm{~g}^{-1}$. The $\mathrm{GeP}_{5}$ electrode in KFSI electrolyte also shows superior rate performance (Fig. 2B), delivering 721.8, 595.2, 503.7, 438.5, 343.9, and $284.2 \mathrm{~mA} \mathrm{~h} \mathrm{~g}^{-1}$ at the current densities of 20,50,100, 200, 500, and $1000 \mathrm{~mA} \mathrm{~g}^{-1}$, respectively. In order to further improve the electrochemical performance of the $\mathrm{GeP}_{5}$ electrodes, fluoroethylene carbonate (FEC) was adopted as an additive, inspired by its application in sodium ion batteries (SIBs) $)^{17,34-36}$. With the addition of 5 wt. \% FEC to both KFSI-EC/DEC and $\mathrm{KPF}_{6}-$ EC/DEC electrolytes, however, the specific capacity dropped quickly after a few cycles at 50 $\mathrm{mA} \mathrm{g}^{-1}$, and the capacity was almost zero at high current densities. To further investigate the effect of FEC additive in the electrolyte, we employed density functional theory (DFT) calculations to obtain the solvation energies in order to understand the interaction between $\mathrm{Na}^{+} / \mathrm{K}^{+}$and the solvent in the electrolyte. The solvation energy in the electrolyte without FEC is notably smaller $(0.305 \mathrm{eV})$ than that in the electrolyte with FEC (1.281 eV) for PIBs (Fig. 2C), indicating that the interaction between $\mathrm{K}^{+}$and the solvent is weaker in the electrolyte without FEC, and therefore, the $\mathrm{K}$ ion diffusion in the electrolyte and the desolvation could be easier, which makes the following reaction with $\mathrm{GeP}_{5}$ easier than in the electrolyte with FEC. Based on 
the DFT calculations (solvation energy), it is apparent that FEC as an additive in PIBs has a negative effect on the electrochemical performance. This seems to be in conflict with the reported effect of FEC as additive in SIBs. We have also calculated the solvation energies in electrolyte for sodium ion batteries. As can be seen in Fig. S1, the solvation energy increased from $0.395 \mathrm{eV}$ to $0.632 \mathrm{eV}$ with the addition of FEC, although the solvation energy in SIBs with FEC $(0.632 \mathrm{eV})$ is much smaller than that in PIBs with FEC $(1.281 \mathrm{eV})$. Also, the FEC, based on reported data ${ }^{17,37-39}$, can help to form a uniform and stable SEI layer on the surface of the electrode, which leads to enhanced cycling performance.

The galvanostatic discharge/charge profiles of $\mathrm{GeP}_{5}$ electrodes for the first three cycles in $\mathrm{KPF}_{6}$ and KFSI electrolytes at $50 \mathrm{~mA} \mathrm{~g}^{-1}$ are shown in Fig. S2. A large irreversible capacity can be found in the first cycle, especially in $\mathrm{KPF}_{6}$ electrolyte, leading to a lower initial $\mathrm{CE}$ than that in KFSI electrolyte. After the addition of FEC (Fig. S3), the $\mathrm{GeP}_{5}$ electrode also exhibited a similar large irreversible capacity in the initial discharge process. This is probably due to greater reduction of the electrolyte to form the SEI, or the excess fluoride may have reacted with the electrode or the potassium metal in the $\mathrm{KPF}_{6}$ electrolyte ${ }^{32}$. To further demonstrate the possible side reactions, the $\mathrm{CEs}$ of the $\mathrm{GeP}_{5}$ electrodes in various electrolytes are presented in Fig. S4. In the following cycles, the CE for KFSI electrolyte stabilized at around 98.5\%, but the CE in $\mathrm{KPF}_{6}$ electrolyte was maintained at $\sim 95 \%$ after 50 cycles. With the FEC additive, the $\mathrm{CE}$ of the $\mathrm{GeP}_{5}$ electrodes was below $85 \%$. These results further suggest that the excess side reactions may have occurred due to the high fluoride concentration in the electrolyte, which may stimulate side reactions during the potassiation process.

In-operando transmission X-ray microscopy (TXM) measurements of the $\mathrm{GeP}_{5}$ used as anode in LIBs were also carried out to reveal the morphological changes induced by the Li storage 
reactions during cycling. It can be observed that, during lithiation and delithiation processes (Fig. $\mathrm{S} 5)$, the $\mathrm{GeP}_{5}$ particle undergoes a similar two-stage mechanism ${ }^{31}$, with this leading to partial particle pulverisation (Fig. S6). It can be imagined that more severe pulverisation and irreversible volume variations will occur when the $\mathrm{GeP}_{5}$ is reacting with $\mathrm{K}$, which has a much larger ionic radius $\left(1.38 \AA\right.$ for $\mathrm{K}^{+}$vs. $0.76 \AA$ for $\left.\mathrm{Li}^{+}\right)$. In order to achieve stable cyclability of the electrodes, besides employing KFSI salt to regulate the formation of the SEI layer, a conductive matrix or void space (porous structure) must be introduced to buffer the mechanical stress induced by the vigorous volume variation of $\mathrm{GeP}_{5}$ during potassiation/depotassiation in order to avoid pulverisation and maintain the integrity of the electrode,. Herein, we mixed ball-milled $\mathrm{GeP}_{5}$ with carbon black, and long-term cycling was conducted at a current density of $500 \mathrm{~mA} \mathrm{~g}^{-1}$ with suitable amounts of carbon (Fig. 2D). After activation during the initial cycle at a current density of $50 \mathrm{~mA} \mathrm{~g}^{-1}$, the $\mathrm{GeP}_{5}$ electrode in KFSI electrolyte exhibited superior stable cycling performance and still maintained $213.7 \mathrm{~mA} \mathrm{~h} \mathrm{~g}^{-1}$ after 2000 cycles. In $\mathrm{KPF}_{6}$ electrolyte, however, the capacity was relatively stable in the first 220 cycles, but it showed severe fluctuations afterwards. Fig. S7 presents representative time-voltage profiles corresponding to certain cycles. The $\mathrm{GeP}_{5}$ electrode in $\mathrm{KPF}_{6}$ electrolyte maintained a stable round-trip performance up to 220 cycles. Afterwards, the voltage fluctuated and required a relatively longer time than normal discharge/charge time intervals. To understand the problem and to optimize the performance of the battery, the cells were cycled in $\mathrm{KPF}_{6}$ electrolyte in different voltage ranges with low cut-off potentials of $0.1 \mathrm{~V}$ and $0.2 \mathrm{~V}$, respectively. As can be observed in Fig. S8, the electrode suffered from capacity fluctuations after around 600 cycles in the voltage range of $0.1-3 \mathrm{~V}$. In contrast, by slightly increasing the low voltage cut-off to $0.2 \mathrm{~V}$, excellent cycling performance could be obtained, even after 1500 cycles, but there is a sacrifice, relatively low specific capacity due to 
the higher cut-off potential. It can be observed that the voltage fluctuated and was retained for a relatively longer time than normal discharge/charge time intervals for the cells in $\mathrm{KPF}_{6}$ electrolyte between 360-380 cycles within the voltage range of 0.1-3 V (Fig. S9). These results suggest that the cycling performance of the electrode can be improved by either using a higher cut-off voltage or employing a suitable electrolyte.

\section{Uniformity and thickness of SEI layer in various electrolytes}

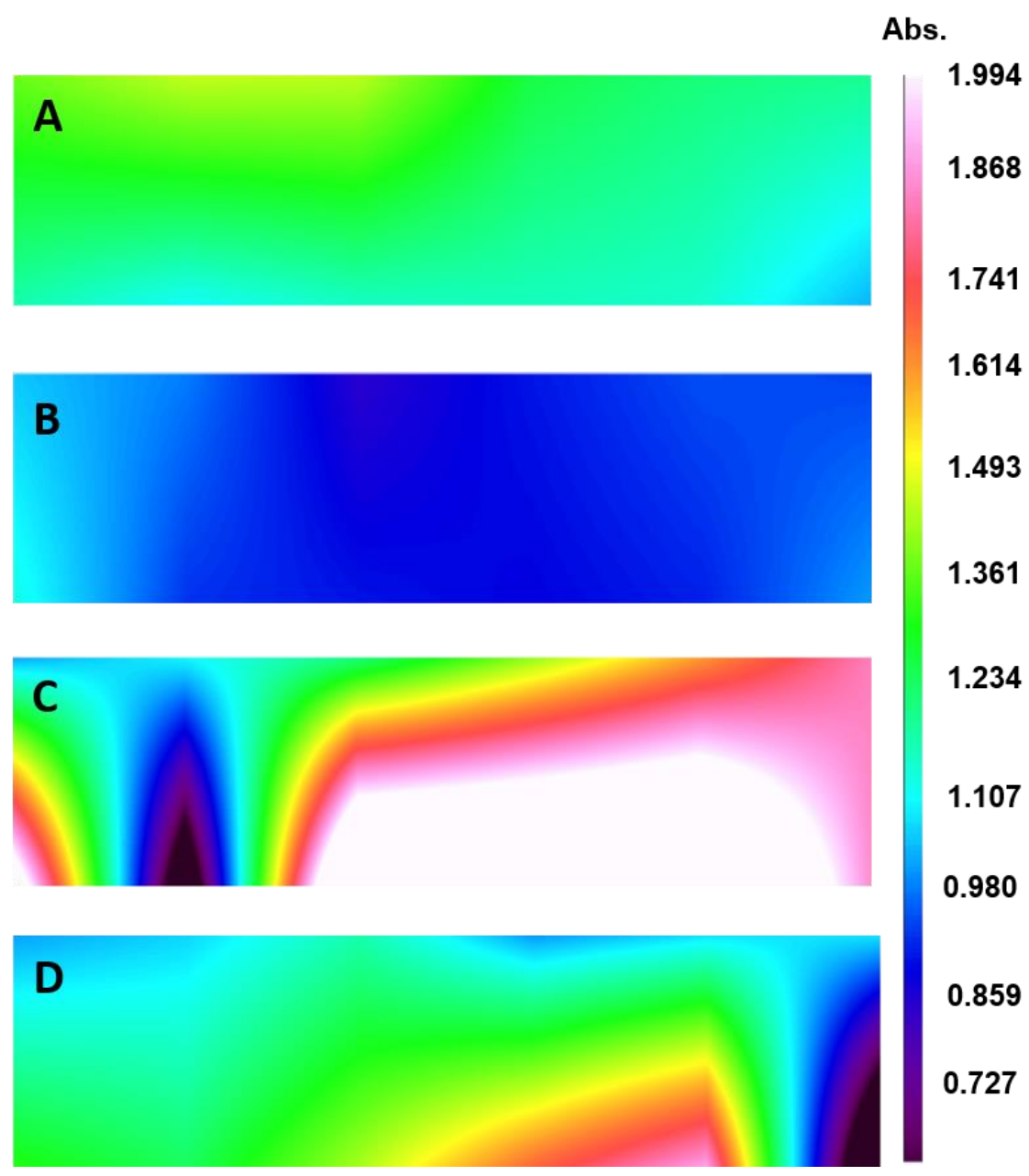

Fig. 3. FTIR mapping of the surfaces of the cycled electrodes. In electrolytes consisting of (A) $\mathrm{KPF}_{6}-\mathrm{EC} / \mathrm{DEC}$, (B) KFSI-EC/DEC, (C) $\mathrm{KPF}_{6}$-EC/DEC with FEC, and (D) KFSI-EC/DEC 
with FEC. (Absorbance (Abs.) ranges were limited from 0.6 to 2.0, with the colour scale based on the corrected absorbance adjusted to the same range.)

Fourier transform infrared (FTIR) spectroscopic mapping was carried out to study the side reactions and distributions of the SEI layer formed in various electrolytes during cycling. The FTIR images were mapped from a set area on the surface of the fresh electrode and the electrode after the $1^{\text {st }}$ cycle, as shown in Fig. 3. The FTIR mapping of the pristine electrode surface is shown in Fig. S10. The $\mathrm{GeP}_{5}$ electrodes in $\mathrm{KPF}_{6}$ and $\mathrm{KFSI}$ electrolyte all exhibited brighter colours (higher Abs. values) than the pristine electrodes, which indicates that the SEI layer could be effectively formed on the surface of the electrode after the $1^{\text {st }}$ cycle. To compare these images, the colour scale based on the corrected absorbance has been adjusted to the same ranges from 0.6 to 2.0. By the semi-quantitative method, areas of contrast can be used to identify the homogeneity and thickness of the SEI layers on the surfaces of the electrodes, with areas of high and low amounts of the imaged chemical entity colour coded as white and black, respectively. As shown in Fig. 3A and 3B, green and blue colour distributions are visible on the surfaces of the electrodes, indicating that uniform SEI layers formed during cycling in $\mathrm{KPF}_{6}$ and KFSI electrolytes. Additionally, the blue colour contrast in KFSI electrolyte suggests that a thinner SEI layer was formed than in the $\mathrm{KPF}_{6}$ electrolyte. To further enhance the image contrast to compare the SEI layers formed in $\mathrm{KPF}_{6}$ and KFSI electrolytes, the detailed colour scale was limited to the range from 0.9 to 1.5 (Fig. S11). The colour mapping reveals that the SEI layer in KFSI electrolyte is more homogeneous than that in $\mathrm{KPF}_{6}$ electrolyte. With the addition of FEC to the electrolytes (Fig. 3C and 3D), the non-uniform colour distributions with white and red contrast 
reveal the formation of thick and inhomogeneous SEI layers on the surfaces of electrodes in electrolytes with FEC.

These results suggest that KFSI as an alternative potassium salt in carbonate electrolytes can effectively help to generate an even and stable SEI layer and possibly avoid excessive sidereactions, resulting in an improved $\mathrm{CE}$, as shown in Fig. S4. In contrast, $\mathrm{KPF}_{6}$ and $\mathrm{FEC}$ additive will stimulate the formation of a relatively thick and uneven SEI layer, leading to low CE and poor cycling performance of the electrodes. Hence, it is proposed that the KFSI electrolyte is beneficial for constructing a uniform and stable SEI film, thus enhancing the cycling stability of the electrodes for PIBs.

\section{In-operando XRD mechanistic study of $\mathrm{GeP}_{5}$ as anode for PIBs}



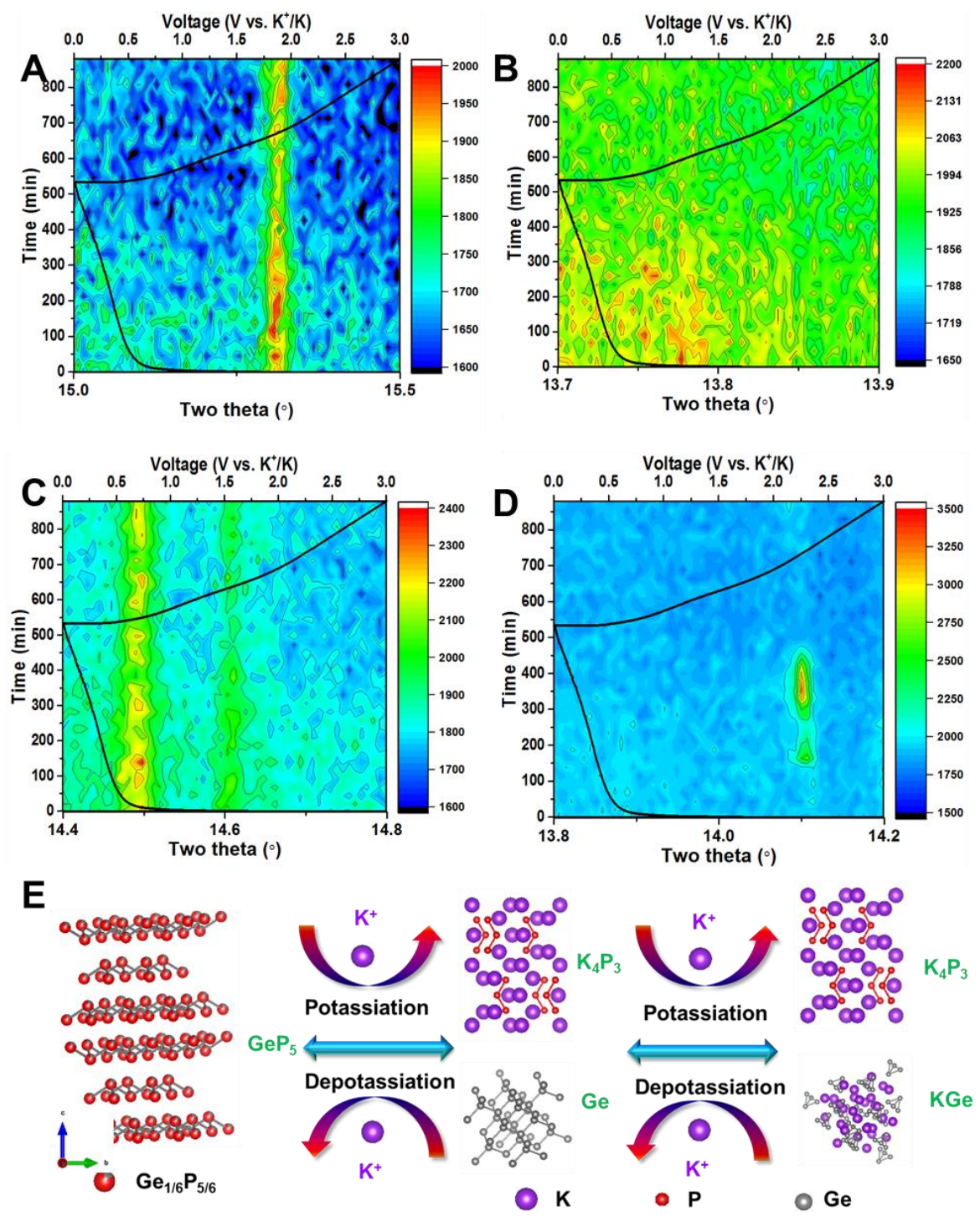

Fig. 4. Electrochemical reaction mechanistic study of GeP5 as anode for PIBs. (A to D)

Contour plots of in-operando synchrotron X-ray powder diffraction with superimposed voltage profiles shown for selected $2 \theta$ ranges of $\mathrm{GeP}_{5}$ as anode for PIBs: (A) $\mathrm{GeP}_{5}(012)$, (B) $\mathrm{K}_{4} \mathrm{P}_{3}(114)$, (C) Ge (201), (D) KGe (215), and (E) schematic illustration of the potassiation/depotassiation process in $\mathrm{GeP}_{5}$ electrode. 
To understand the electrochemical reaction mechanism of $\mathrm{GeP}_{5}$ as anode for PIBs, the mechanistic response of $\mathrm{GeP}_{5}$ anode during cycling within a potential window of $0.01-3.0 \mathrm{~V}$ (vs. $\left.\mathrm{K}^{+} / \mathrm{K}\right)$ was characterised using in-operando synchrotron $\mathrm{XRD}(\lambda=0.68899 \AA)$. The measurements were carried out at the Powder Diffraction Beamline, Australian Synchrotron. The in-operando diffraction data for the $\mathrm{GeP}_{5}$ cell in the first cycle are represented by the contour plots of the operando data in selected $2 \theta$ ranges in Fig. 4A-D. The intensity of the $\mathrm{GeP}_{5}(012)$ reflection at $2 \theta=15.31^{\circ}$ decreased with the discharge process down to $0.01 \mathrm{~V}$ and afterwards increased with the charge process (Fig. 4A), which indicates the consumption of $\mathrm{GeP}_{5}$ phase during discharging (potassiation) and its reversibility during charging (depotassiation). The insufficient reaction of $\mathrm{GeP}_{5}$ is possibly due to the relatively large current density and high loading amount for synchrotron experiments. In the initial discharge process, a peak for the $\mathrm{K}_{4} \mathrm{P}_{3}$ nanoparticle (114) reflection at $2 \theta=13.78^{\circ}$ (Fig. 4B) appears, and its intensity increases. Upon further discharge, the intensity of the Ge reflection at $2 \theta=14.49^{\circ}$ (Fig. 4C) increases to its maximum after discharging to $\sim 0.45 \mathrm{~V}$ and then decreases. After further discharge, the peak for the $\mathrm{KGe}(04-007-1643)$ reflection at $2 \theta=14.07^{\circ}$ (Fig. 4D) appears at around $0.4 \mathrm{~V}$ and remains until full discharge.

According to these results, it can be concluded that the potassiation process for $\mathrm{GeP}_{5}$ could be divided into two stages and described by the following equations:

$$
\begin{gathered}
\mathrm{Ge} P_{5}+20 / 3 K \leftrightarrow 5 / 3 K_{4} P_{3}+G e \\
G e+K \leftrightarrow K G e
\end{gathered}
$$

The reaction mechanism for $\mathrm{GeP}_{5}$ during potassiation/depotassiation is schematically shown in Fig. 4E. In stage I, a conversion reaction occurs, $\mathrm{GeP}_{5}$ decomposes into Ge and $\mathrm{P}$ particles, and 
the $\mathrm{P}$ component reacts with $\mathrm{K}$ to form $\mathrm{K}_{4} \mathrm{P}_{3}$. In stage $\mathrm{II}, \mathrm{Ge}$ alloys with $\mathrm{K}$ to form $\mathrm{KGe}$. Consequently, the synergistic K-storage reactions of K-P phases $\left(\mathrm{K}_{4} \mathrm{P}_{3}\right)$ and $\mathrm{K}-\mathrm{Ge}(\mathrm{KGe})$ alloys during the discharge process can create a mutual buffer mechanism. This buffering effect can alleviate the volume changes during cycling, thus helping to maintain the integrity of the electrode and improve the cycling stability.

\section{Conclusions}

In summary, we have reported a layered alloy compound, $\mathrm{GeP}_{5}$, with high phosphorus concentration as anode material for PIBs. The $\mathrm{GeP}_{5}$ electrode can deliver a high reversible specific capacity over $500 \mathrm{~mA} \mathrm{~h} \mathrm{~g}^{-1}$ and exhibits excellent rate capability $\left(230 \mathrm{~mA} \mathrm{~h} \mathrm{~g}^{-1} \mathrm{at}_{1 \mathrm{~A} \mathrm{~g}^{-}}\right.$ ${ }^{1}$ ) for $\mathrm{K}$ ion storage. The superior electrochemical performance is attributable to the synergistic reaction mechanism (alloying and conversion reactions) during the discharge/charge processes, which greatly buffers volume changes in the electrode. An alternative potassium salt (KFSI) has been demonstrated to promote a uniform, stable SEI layer and allow long cyclability for PIBs. This work suggests the great potential of $\mathrm{GeP}_{5}$ as anode for PIBs and is likely to attract more interest in exploring alloy-based electrode materials with high volumetric capacity in electrochemical energy storage.

\section{Conflicts of interest}

There are no conflicts to declare. 


\section{Acknowledgements}

Financial support provided by the Australian Research Council (ARC) (FT150100109, FT160100251, DP170102406, LE180100141), the National Natural Science Foundation of China (Grant No. 21701030), the Guangdong Province Natural Science Foundation (Grant No. 2017A030310241), and the Innovative Talents Cultivation Project of Outstanding Youth in Guangdong Province (Grant No. 2016KQNCX038) is gratefully acknowledged. Pang is grateful to the financial support from the University of Wollongong through the UIC International Links Grant Scheme 2018. W.C. Zhang would like to thank the University of Wollongong for financial support. The authors are grateful to Prof. Dianwu Zhou at Hunan University for support of the DFT calculation software, Dr. Chuang-Fong Kong of Perkin Elmer, Australia, for her operational support on Fourier transform infrared spectroscopy measurements, and Dr Yeng-Fang Song of NSRRC for her support on the transmission X-ray microscopy experiments. The authors also thank Dr. Tania Silver for performing critical revision of the manuscript.

\section{Supporting Information.}

Additional details on solvation energy calculations, galvanostatic discharge/charge curves, coulombic efficiency, TXM micrographs, time-voltage profiles, long-term cycling tests in various voltage ranges, and FTIR mapping images with different absorbance ranges.

\section{Conflicts of interest}

There are no conflicts to declare.

\section{Corresponding Author}

* Email: wkpang@uow.edu.au (W.P.); 
*Email: wenwuli@hust.edu.cn (W.L.);

*Email: zguo@uow.edu.au (Z.G.)

\section{References}

1 D. Larcher, J. Tarascon. Towards greener and more sustainable batteries for electrical energy storage. Nat. Chem. 7, 19-29 (2015).

2 B. Kang, G. Ceder. Battery materials for ultrafast charging and discharging. Nature 458, 190 (2009).

3 H. Zhang, J. Nai, L. Yu, X. W. D. Lou. Metal-organic-framework-based materials as platforms for renewable energy and environmental applications. Joule 1, 77-107 (2017).

4 A. S. Arico, P. Bruce, B. Scrosati, J.-M. Tarascon, W. Van Schalkwijk. Nanostructured materials for advanced energy conversion and storage devices. Nat. Mater. 4, 366 (2005).

5 M. Li, J. Lu, Z. Chen, K. Amine. 30 Years of Lithium - Ion Batteries. Adv. Mater., 1800561 (2018).

6 C. Hu, X. Chen, Q. Dai, M. Wang, L. Qu, L. Dai. Earth-abundant carbon catalysts for renewable generation of clean energy from sunlight and water. Nano Energy 41, 367-376 (2017).

7 C. Hu, L. Dai. Multifunctional Carbon - Based Metal - Free Electrocatalysts for Simultaneous Oxygen Reduction, Oxygen Evolution, and Hydrogen Evolution. Adv. Mater. 29, 1604942 (2017).

8 H. B. Wu, X. W. D. Lou. Metal-organic frameworks and their derived materials for electrochemical energy storage and conversion: Promises and challenges. Sci. Adv. 3, eaap9252 (2017).

9 J. B. Goodenough, K.-S. Park. The Li-ion rechargeable battery: a perspective. J. Am. Chem. Soc. 135, 1167-1176 (2013).

10 J. Xie, L. Liao, Y. Gong, Y. Li, F. Shi, A. Pei, J. Sun, R. Zhang, B. Kong, R. Subbaraman. Stitching h-BN by atomic layer deposition of $\mathrm{LiF}$ as a stable interface for lithium metal anode. Sci. Adv. $\mathbf{3}$, eaao3170 (2017).

11 Y. Wang, R. Zhang, Y.-c. Pang, X. Chen, J. Lang, J. Xu, C. Xiao, H. Li, K. Xi, S. Ding. Carbon@ titanium nitride dual shell nanospheres as multi-functional hosts for lithium sulfur batteries. Energy Storage Materials 16, 228-235 (2019).

12 Y. Liu, X.-Y. Yu, Y. Fang, X. Zhu, J. Bao, X. Zhou, X. W. D. Lou. Confining SnS2 Ultrathin Nanosheets in Hollow Carbon Nanostructures for Efficient Capacitive Sodium Storage. Joule 2, 725735 (2018).

13 I. Sultana, M. M. Rahman, Y. Chen, A. M. Glushenkov. Potassium - Ion Battery Anode Materials Operating through the Alloying-Dealloying Reaction Mechanism. Adv. Funct. Mater. (2017).

14 A. Eftekhari, Z. Jian, X. Ji. Potassium secondary batteries. ACS Appl. Mater. Interfaces 9, 4404-4419 (2016).

15 P.-F. Wang, H.-R. Yao, X.-Y. Liu, Y.-X. Yin, J.-N. Zhang, Y. Wen, X. Yu, L. Gu, Y.-G. Guo. $\mathrm{Na}+$ /vacancy disordering promises high-rate Na-ion batteries. Sci. Adv. 4, eaar6018 (2018).

16 N. Yabuuchi, K. Kubota, M. Dahbi, S. Komaba. Research development on sodium-ion batteries. Chem. Rev. 114, 11636-11682 (2014).

17 M. D. Slater, D. Kim, E. Lee, C. S. Johnson. Sodium - ion batteries. Adv. Funct. Mater. 23, 947-958 (2013). 

(2015).

19 K. Lei, C. Wang, L. Liu, Y. Luo, C. Mu, F. Li, J. Chen. A Porous Network of Bismuth Used as the Anode Material for High - Energy - Density Potassium - Ion Batteries. Angew. Chem. 130, 47774781 (2018).

20 Q. Zhang, J. Mao, W. K. Pang, T. Zheng, V. Sencadas, Y. Chen, Y. Liu, Z. Guo. Boosting the Potassium Storage Performance of Alloy - Based Anode Materials via Electrolyte Salt Chemistry. Adv. Energy Mater., 1703288 (2018).

21 J. Zhao, X. Zou, Y. Zhu, Y. Xu, C. Wang. Electrochemical Intercalation of Potassium into Graphite. Adv. Funct. Mater. (2016).

22 J. Yang, Z. Ju, Y. Jiang, Z. Xing, B. Xi, J. Feng, S. Xiong. Enhanced Capacity and Rate Capability of Nitrogen/Oxygen Dual - Doped Hard Carbon in Capacitive Potassium - Ion Storage. Adv. Mater. (2017).

23 C. Chen, Z. Wang, B. Zhang, L. Miao, J. Cai, L. Peng, Y. Huang, J. Jiang, Y. Huang, L. Zhang. Nitrogen-rich hard carbon as a highly durable anode for high-power potassium-ion batteries. Energy Storage Materials 8, 161-168 (2017).

24 W. Zhang, J. Mao, S. Li, Z. Chen, Z. Guo. Phosphorus-based alloy materials for advanced potassium-ion battery anode. J. Am. Chem. Soc. 139, 3316-3319 (2017).

25 P. Lou, Z. Cui, Z. Jia, J. Sun, Y. Tan, X. Guo. Monodispersed carbon-coated cubic NiP2 nanoparticles anchored on carbon nanotubes as ultra-long-life anodes for reversible lithium storage. ACS Nano 11, 3705-3715 (2017).

26 Y. Lu, P. Zhou, K. Lei, Q. Zhao, Z. Tao, J. Chen. Selenium Phosphide (Se4P4) as a New and Promising Anode Material for Sodium - Ion Batteries. Adv. Energy Mater. 7 (2017). Y. Kim, Y. Kim, A. Choi, S. Woo, D. Mok, N. S. Choi, Y. S. Jung, J. H. Ryu, S. M. Oh, K. T. Lee. Tin Phosphide as a Promising Anode Material for Na - Ion Batteries. Adv. Mater. 26, 4139-4144 (2014). X. Fan, J. Mao, Y. Zhu, C. Luo, L. Suo, T. Gao, F. Han, S. C. Liou, C. Wang. Superior Stable Self Healing SnP3 Anode for Sodium - Ion Batteries. Adv. Energy Mater. 5 (2015). W. Zhang, J. Mao, W. K. Pang, Z. Guo, Z. Chen. Large-scale synthesis of ternary Sn5SbP3/C composite by ball milling for superior stable sodium-ion battery anode. Electrochim. Acta 235, 107113 (2017).

30 J. Qian, Y. Xiong, Y. Cao, X. Ai, H. Yang. Synergistic Na-storage reactions in Sn4P3 as a highcapacity, cycle-stable anode of Na-ion batteries. Nano Lett. 14, 1865-1869 (2014).

31 W. Li, H. Li, Z. Lu, L. Gan, L. Ke, T. Zhai, H. Zhou. Layered phosphorus-like GeP 5: a promising anode candidate with high initial coulombic efficiency and large capacity for lithium ion batteries. Energy Environ. Sci. 8, 3629-3636 (2015).

32 N. Xiao, W. D. McCulloch, Y. Wu. Reversible Dendrite-Free Potassium Plating and Stripping Electrochemistry for Potassium Secondary Batteries. J. Am. Chem. Soc. 139, 9475-9478 (2017).

33 W. Zhang, W. K. Pang, V. Sencadas, Z. Guo. Understanding High-Energy-Density Sn4P3 Anodes for Potassium-Ion Batteries. Joule 2(8), 1534-1547 (2018).

34 J. Qian, X. Wu, Y. Cao, X. Ai, H. Yang. High capacity and rate capability of amorphous phosphorus for sodium ion batteries. Angew. Chem. 125, 4731-4734 (2013).

35 Y. Kim, Y. Park, A. Choi, N. S. Choi, J. Kim, J. Lee, J. H. Ryu, S. M. Oh, K. T. Lee. An amorphous red phosphorus/carbon composite as a promising anode material for sodium ion batteries. Adv. Mater. 25, 3045-3049 (2013).

36 W. Li, S. Hu, X. Luo, Z. Li, X. Sun, M. Li, F. Liu, Y. Yu. Confined Amorphous Red Phosphorus in MOF - Derived N - Doped Microporous Carbon as a Superior Anode for Sodium - Ion Battery. Adv. Mater. 29 (2017). 
Q. Wang, W. Zhang, C. Guo, Y. Liu, C. Wang, Z. Guo. In Situ Construction of 3D Interconnected FeS@ Fe3C@ Graphitic Carbon Networks for High - Performance Sodium - Ion Batteries. Adv. Funct. Mater. 27, 1703390 (2017).

38 W. Zhang, J. Mao, W. K. Pang, X. Wang, Z. Guo. Creating fast ion conducting composites via insitu introduction of titanium as oxygen getter. Nano Energy 49, 549-554 (2018).

39 Z. Tu, S. Choudhury, M. J. Zachman, S. Wei, K. Zhang, L. F. Kourkoutis, L. A. Archer. Designing artificial solid-electrolyte interphases for single-ion and high-efficiency transport in batteries. Joule $\mathbf{1}$, 394-406 (2017).

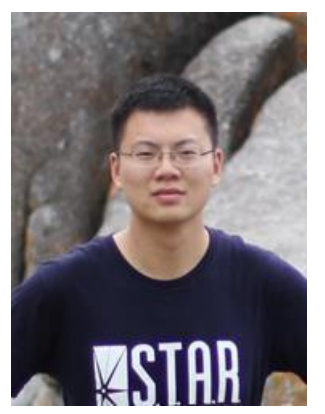

Wenchao Zhang earned his B.S. and Master's degrees in Materials Science and Engineering at the Central South University, PR China in 2012 and 2015, respectively. He is a $\mathrm{Ph} . \mathrm{D}$. candidate under the supervision of Prof. Zaiping Guo at the School of Mechanical, Materials, Mechatronics and Biomedical Engineering, University of Wollongong, Australia. He is currently a visiting scholar under the supervision of Prof. Liming Dai in the Department of Macromolecular Science and Engineering, Case Western Reserve University, USA. His current research interests are focused on the design and application of alloy-based anode materials for metal-ion batteries.

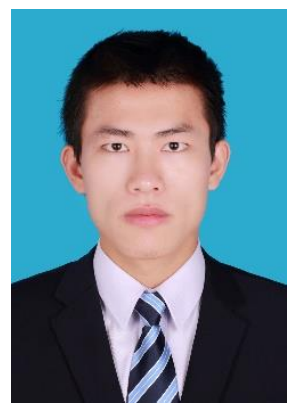

Zhibin Wu received his B.S. degree in 2013 and M.S. degree in 2016 from Central South University. At present, he is pursuing his $\mathrm{PhD}$ degree in the Institute for Superconducting \& Electronic Materials Institute (ISEM) at the University of Wollongong. His current research is focused on investigating the mechanisms and performance of alkali metal-ion batteries based on transition metal chalcogenides, and the synchrotron techniques relating to X-ray Absorption Spectroscopy and Powder Diffraction. 


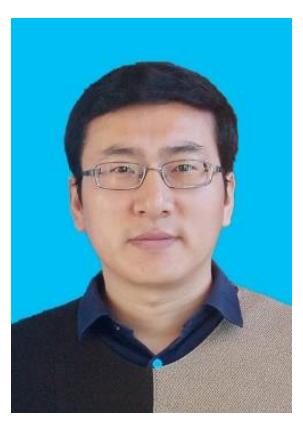

Jian Zhang obtained his Ph.D. degree from Hunan University in 2009. After graduation, he joined Changsha University of Science and Technology. He is currently an associate professor in the College of Automotive and Mechanical Engineering, Changsha University of Science and Technology. His work is focused on first-principles calculations and the design of energy storage materials, including hydrogen storage and rechargeable batteries.

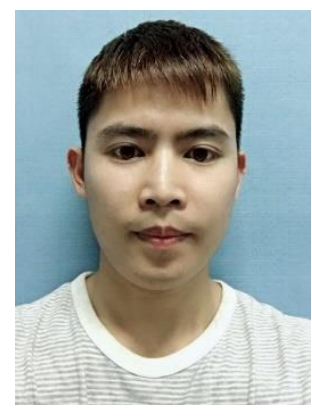

Guoping Liu received his B.S. degree from the East China University of Technology in 2016. Now he is a Master's degree candidate of the School of Materials and Energy, Guangdong University of Technology (GDUT). His research is concentrated on developing high-performance anode materials for rechargeable alkali metal ion batteries.

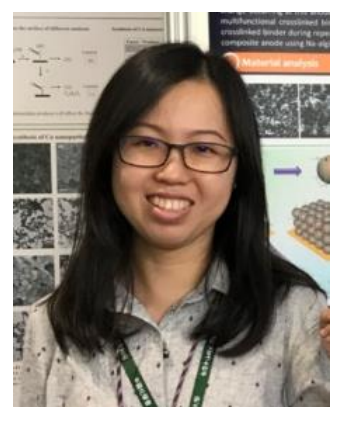

Nai-Hsuan Yang is a postdoctoral researcher and leader of the Energy Storage Group at the Materials Chemistry Laboratory at National Taiwan University. She received her Bachelor's and Master's degrees in Chemical Engineering from Chung Yuan University in 2005 and 2007, respectively. She completed her $\mathrm{PhD}$ at National Taiwan University. Her research interests are focused on silicon carbon composites, amorphous carbon, and silicon anodes with polymeric binders for LIBs, the applications of $\mathrm{Si}$ as anode in the next generation of LIBs, and the application of TXM to detect morphology changes of electrodes under operation. 


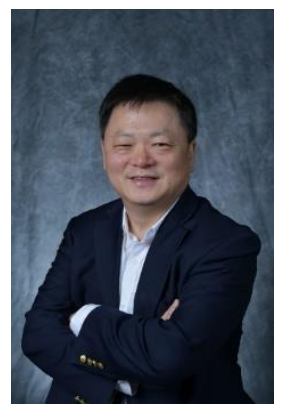

Ru-Shi Liu is a Professor of Department of Chemistry, National Taiwan University, Taiwan. He received his Bachelor's degree in Chemistry from Soochow University, Taiwan, in 1981. He was awarded his Master's degree in nuclear science from the National Tsing Hua University, Taiwan, in 1983. He obtained two Ph.D. degrees in Chemistry from National Tsing Hua University, Taiwan, in 1990 and from the University of Cambridge, England, in 1992. His research is focused on the developments of photo-conversion materials, including light-emitting diode phosphors, energy conversion and storage devices, and nanobio therapies

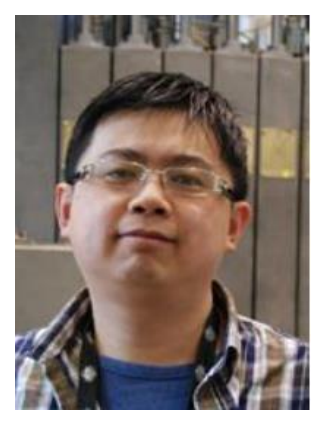

Wei Kong Pang received his Ph.D. in Applied Physics from Curtin University, Western Australia in 2011. After completion, he was first appointed as a postdoctoral research fellow in the Department of Chemistry at the National Taiwan University and the Department of Materials Engineering at Tatung University, Taiwan. In 2013 he joined the University of Wollongong and the Australian Nuclear Science and Technology Organisation as a postdoctoral research fellow. Recently he was awarded an Australian Research Council (ARC) Future Fellowship and is currently a senior research fellow at the Institute for Superconducting and Electronic Materials (ISEM), University of Wollongong.

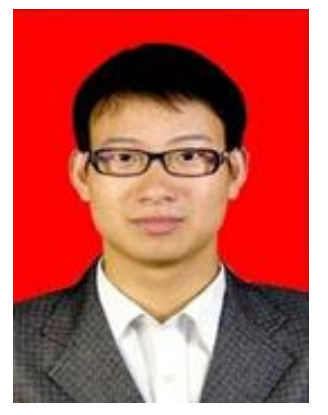

Wenwu Li received his Ph.D. degree in Materials Science and Engineering from Huazhong University of Science and Technology in 2016. Then, he worked as an associate professor in the School of Materials and Energy, Guangdong University of Technology, Guangzhou, China. He is currently a 
visiting scholar in the School of Materials Science and Engineering, Georgia Institute of Technology, USA. His current research interests are focused on designing novel P-based anode materials with high-security for metal ion batteries and uncovering the fundamental structureperformance relationships behind high metal ion storage performance and the mechanisms involved.

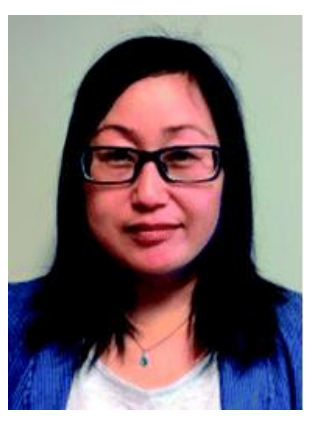

Guo Zaiping obtained her Ph.D. from the University of Wollongong in 2003. After her postdoctoral research with Prof. Huakun Liu at the Institute for Superconducting and Electronic Materials (ISEM), University of Wollongong, she joined the Faculty of Engineering in 2008. She is currently a senior professor and ARC Queen Elizabeth II (QEII) Fellow in the School of Mechanical, Materials \& Mechatronic Engineering and the Institute for Superconducting \& Electronic Materials, University of Wollongong. Her work is focused on practical applications of various nanomaterials as electrode materials for energy storage or conversion technologies, including rechargeable batteries, hydrogen storage, and supercapacitors. 


\section{Supporting Information}

\section{Unraveling the effect of salt chemistry on long-durability high-phosphorus-concentration anode for potassium ion batteries}

Wenchao Zhang ${ }^{1}$, Zhibin $\mathrm{Wu}^{1}$, Jian Zhang ${ }^{3}$, Guoping Liu ${ }^{2}$, Nai-Hsuan Yang ${ }^{4}$, Ru-Shi Liu ${ }^{4}$, Wei Kong Pang ${ }^{1 *}$, Wenwu $\mathrm{Li}^{2 *}$, Zaiping Guo ${ }^{1 *}$

[1] Institute for Superconducting \& Electronic Materials, School of Mechanical, Materials, Mechatronics \& Biomedical Engineering, Faculty of Engineering and Information Sciences, University of Wollongong, NSW 2500, Australia.

[2] School of Materials and Energy, Guangdong University of Technology, No. 100 Waihuan Xi Road, Guangzhou Higher Education Mega Center, Guangzhou 510006, P. R. China.

[3] College of Automotive and Mechanical Engineering, Changsha University of Science and Technology, Changsha 410114, China

[4] Department of Chemistry, National Taiwan University, Taipei 106, Taiwan

*Corresponding author. Email: wkpang@uow.edu.au (W.P.); wenwuli@ hust.edu.cn (W.L.); zguo@uow.edu.au (Z.G.) 


\section{Experimental Section}

Preparation of $\mathrm{GeP}_{5}$ powder:

$\mathrm{GeP}_{5}$ powder was obtained by a high-energy ball-milling (HEBM, Fritsch Pulverisette-6. Germany) process using commercial Ge and red phosphorus as the raw materials in the mole ratio of Ge: $\mathrm{P}=1: 5$. The HEBM was conducted at $500 \mathrm{rpm}$ for $10 \mathrm{~h}$, and the elemental mixture was milled using ball-mill beads $10 \mathrm{~mm}$ in diameter with a powder-to-ball weight ratio of 1:20. All sample storage and handling were performed in an Ar filled glove box (MBraun Unilab).

\section{Materials characterization}

The crystal structures of the as-prepared powders were characterized by powder X-ray diffraction (XRD) on a GBC MMA diffractometer with a $\mathrm{Cu} \mathrm{K} \alpha$ source at a scanning rate of $1^{\circ}$ $\min ^{-1}$. The morphology of the as-synthesized powders was characterized on a JEOL JSM7500FA field-emission scanning electron microscope (FESEM) and a JEOL ARM-200F aberration-corrected transmission electron microscope (TEM). A customized CR2032 coin cell was made for use in synchrotron X-ray powder diffraction (SXRPD) experiments, and the details of cell assembly can be found elsewhere ${ }^{1}$. The cell was galvanostatically discharged and charged over $0.01-3 \mathrm{~V}$ vs. $\mathrm{K}^{+} / \mathrm{K}$. Synchrotron X-ray powder diffraction (SXRPD) experiments were conducted on the Powder Diffraction beamline at the Australian Synchrotron, where the collection time for each data point was 180 seconds and the time gap between each collection was 7.5 minutes during discharging and charging using a MYTHEN microstrip detector. The 
wavelength of synchrotron radiation was determined to be $0.68899 \AA$ using $\mathrm{LaB}_{6}$ (Standard Reference Material SRM 660b).

Fourier-transform infrared (FTIR) maps were collected using a PerkinElmer Spotlight 400 equipped with a mercury cadmium tellurium (MCT) detector, which consists of single point (point mode) and 16-array (image mode) detectors all in one. Maps of $2 \times 8$ points $(\sim 200 \times 100$ $\mu \mathrm{m}^{2}$ area) in reflectance mode were acquired at $4 \mathrm{~cm}^{-1}$ resolution with 16 scans per pixel from 4000 to $400 \mathrm{~cm}^{-1}$. The drop down average true range (ATR) is a Ge crystal $100 \mu \mathrm{m}$ in diameter. Principal component analysis (PCA) is one of the most widely used methods for data reduction and exploratory analysis. In this work, PCA analyses of the selected FTIR regions corresponding to the $400-4000 \mathrm{~cm}^{-1}$ range were obtained to reveal the uniformity of the functional groups and solid electrolyte interphase (SEI) layer.

\section{Theoretical calculations}

The calculations were performed based on the density functional theory (DFT) approach ${ }^{2}$ using the $\mathrm{DMol}^{3}$ package. The effects of exchange correlation interaction were treated according to the Perdew-Burke-Ernzerhof generalized-gradient approximation (GGA-PBE) ${ }^{3}$. All-electron KohnSham wave functions were expanded in a double numerical basis with polarized orbital (DNP) ${ }^{4}$. Sampling of the irreducible wedge of the Brillouin zone was performed with a regular Monkhorst-Pack grid of special k-points ${ }^{5}$. The convergence criteria for relaxation were $1.0 \times 10^{-}$

${ }^{5} \mathrm{Ha}, 0.002 \mathrm{Ha} / \AA$, and $0.005 \AA$ for the energy, gradient, and atomic displacement, respectively.

The ion solvation is directly related to the strength of the ion-solvent interaction that governs the solvation structure. In addition, it plays an important role in determining the energetics of ion intercalation from the liquid electrolyte into the battery electrodes, since the desolvation is 
required for intercalation ${ }^{6,7}$. The ion solvation energies were approximated from the binding energy of the $\mathrm{X}^{+}(\mathrm{Y})$ clusters, where $\mathrm{X}=\mathrm{K}^{+}$or $\mathrm{Na}^{+}$, and $\mathrm{Y}=\mathrm{EC}+\mathrm{DEC}$ or $\mathrm{EC}+\mathrm{DEC}+\mathrm{FEC}$. In particular

$$
\Delta E_{s o l}=E_{X^{+}}+E_{(Y)}-E_{X^{+}(Y)}
$$

where $E_{X^{+}}, E_{(Y)}$, and $E_{X^{+}(Y)}$ are the energies of the $\mathrm{X}^{+}$in vacuum, and the $(\mathrm{Y})$ and $\mathrm{X}^{+}(\mathrm{Y})$ clusters, respectively ${ }^{8}$.

\section{TXM characterization}

In-operando transmission X-ray microscopy (TXM) measurements of the $\mathrm{GeP}_{5}$ used as anode in LIBs were carried out at the 01B1 beamline of the National Synchrotron Radiation Research Center (NSRRC) in Hsinchu City, Taiwan. The light source operates with photon energy ranging between 8 and $11 \mathrm{keV}$. The $\mathrm{X}$-rays passing through the tested coin cell go through a zone plate optical system and then a phase ring to form the image.

The electrochemical testing was carried out by using a 2032-type coin cell with a hole in the stainless steel disk, top, and bottom cap. The working electrode consisted of $\mathrm{GeP}_{5}$, conductive additives, and binder in the weight ratio of 40:40:20. The conductive additives included graphitic flakes (KS6, $3 \mathrm{~lm}$, Timcal) and carbon black (Super P, $40 \mathrm{~nm}$, Timcal). Lithium polyacrylate was chosen as binder because of its multifunctional nature and good properties for high-voltage Liion batteries. The $\mathrm{GeP}_{5}$ electrode was prepared by coating the slurry on a disk. Once the film dried, the layer was removed from the substrate, creating a free-standing film. The free-standing electrode was further dried under vacuum overnight to remove residual solvent. The counter electrode was Li foil, which is mostly transparent to X-rays. The electrolyte was a $1 \mathrm{M}$ solution of $\mathrm{LiPF}_{6}$ in ethylene carbonate/ethyl methyl carbonate (1:2 vol. \%). The covers of both sides of 
the cell were sealed with Kapton tapes in order to allow the X-ray beam to pass through the cell. The cell-assembly processes were conducted in a glove box, where oxygen and water levels were kept lower than 1 ppm.

The charge/discharge tests were carried out in constant current (CC) mode within the voltage window from $3 \mathrm{~V}$ down to $0.005 \mathrm{~V}$ under the current density of $0.3 \mathrm{~A} \mathrm{~g}^{-1}$. All the potentials reported herein are referenced to $\mathrm{Li} / \mathrm{Li}^{+}$.

\section{Electrochemical measurements}

Electrodes were fabricated using a slurry-coating method. The synthesized materials $\left(\mathrm{GeP}_{5}\right)$ were mixed with Super P carbon black and poly(acrylic acid) (PAA) in the weight ratio of 3:5:2, respectively. Deionized (DI) water was used as the dispersing agent. Then, the slurry was coated on copper foil and dried in a vacuum oven at $80{ }^{\circ} \mathrm{C}$ overnight. Coin-type (CR2032) cells were assembled in an argon-filled glove box with oxygen and water content lower than $0.1 \mathrm{ppm}$. K half-cells were assembled with potassium metal and $0.8 \mathrm{M} \mathrm{KPF}_{6}$ or $1 \mathrm{M} \mathrm{KFSI}$ in an EC/DEC solution $(\mathrm{EC}: \mathrm{DEC}=1: 1, \mathrm{v} / \mathrm{v})$ as electrolyte. FEC (5 wt. \%) as an additive was added into the electrolyte for comparison purposes. The cells were galvanostatically charged-discharged between 0.01 and $3.0 \mathrm{~V}$ versus $\mathrm{K} / \mathrm{K}^{+}$at various current densities on a Neware tester. 

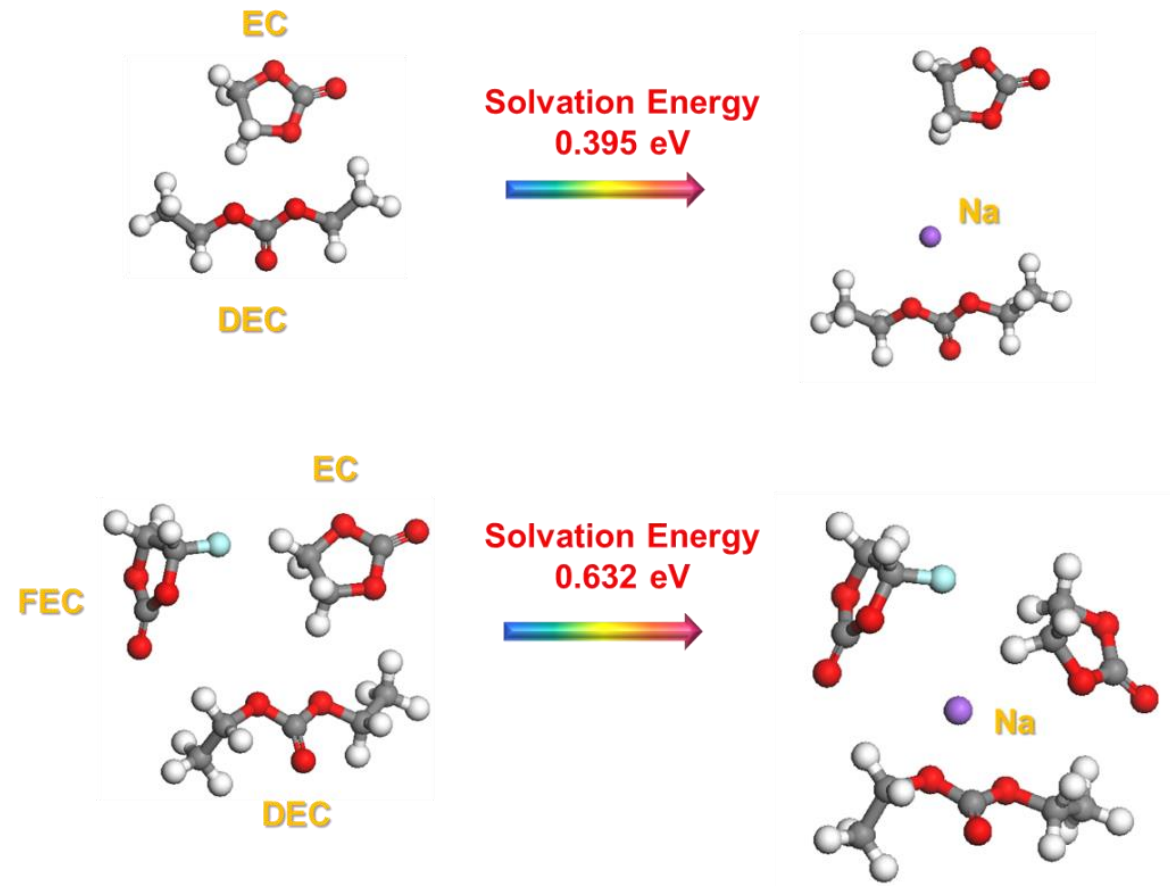

Fig. S1. Solvation energies estimated from the binding energy of the $\mathrm{Na}^{+}(\mathrm{Y})$ clusters, where $\mathrm{Y}=$ $\mathrm{EC}+\mathrm{DEC}$ or $\mathrm{EC}+\mathrm{DEC}+\mathrm{FEC}$. 

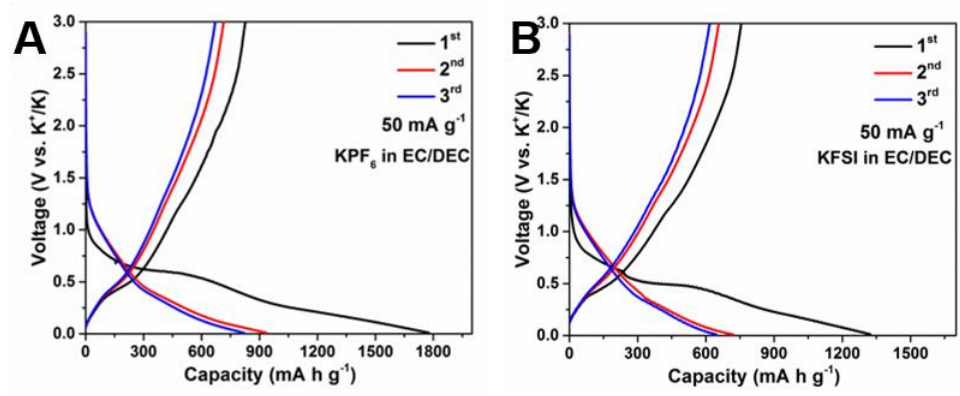

Fig. S2. Galvanostatic discharge and charge profiles for the first 3 cycles of the electrode in (A) $\mathrm{KPF}_{6}$ and (B) KFSI electrolyte at $50 \mathrm{~mA} \mathrm{~g}^{-1}$. 

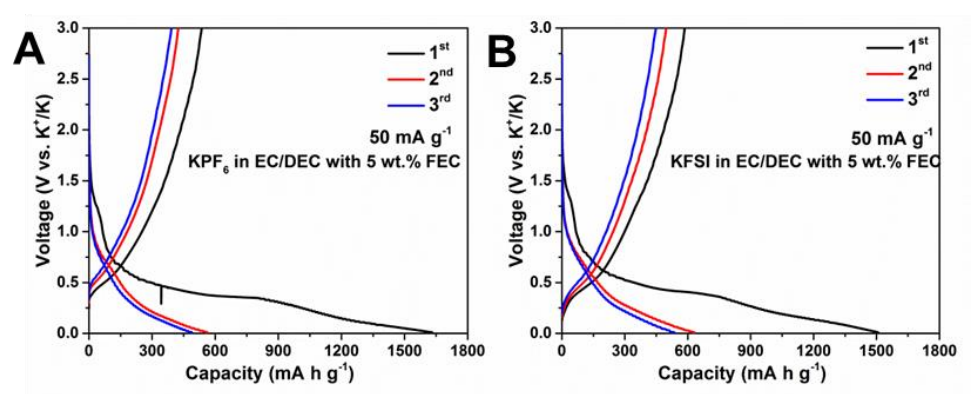

Fig. S3. Galvanostatic discharge and charge profiles for the first 3 cycles of the electrode in (A) $\mathrm{KPF}_{6}$ and (B) KFSI electrolyte with 5 wt. \% FEC at $50 \mathrm{~mA} \mathrm{~g}^{-1}$. 


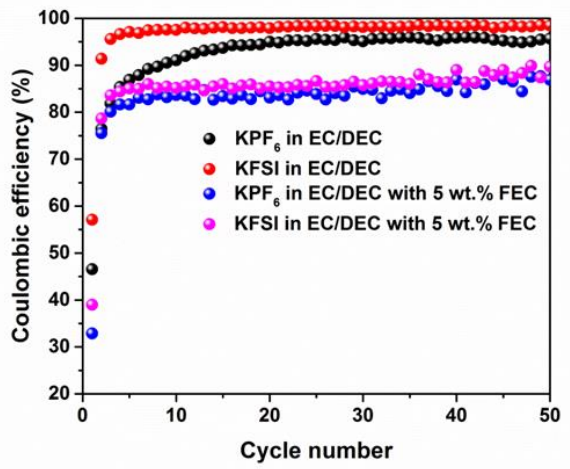

Fig. S4. Coulombic efficiency of the $\mathrm{GeP}_{5}$ electrode in various electrolytes. 


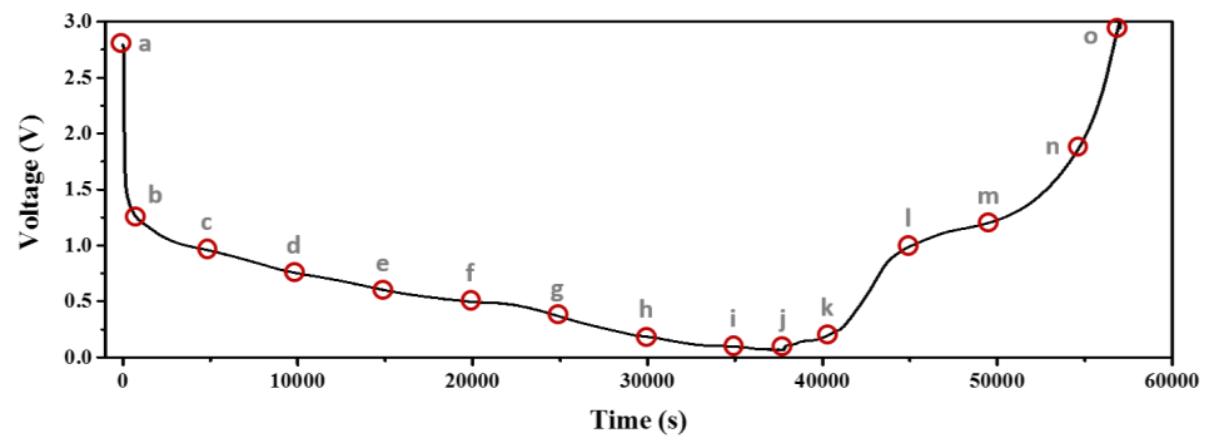

Fig. S5. The first lithiation/de-lithiation process in $\mathrm{GeP}_{5}$ electrode. 

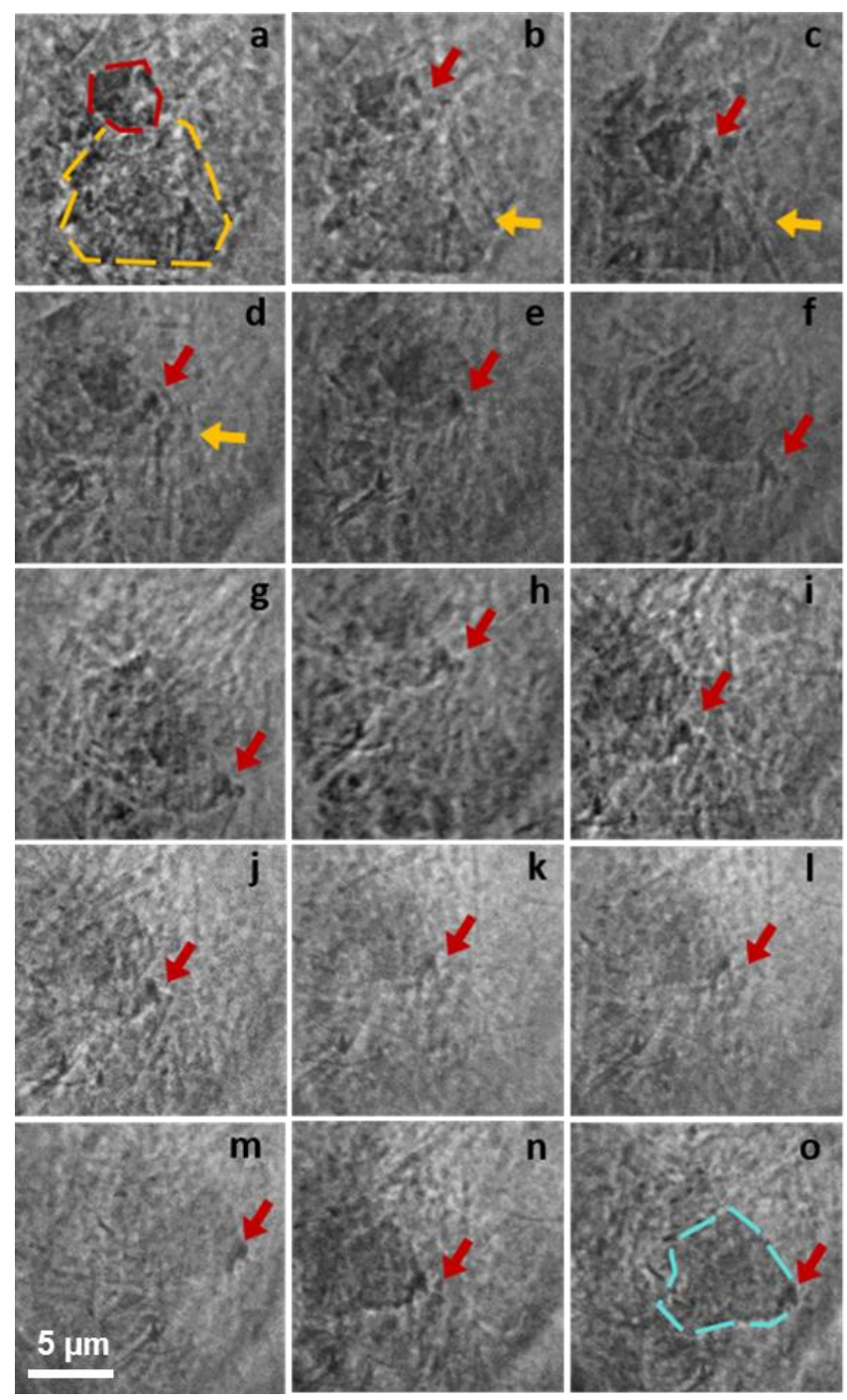

Fig. S6. TXM micrographs of selected particles.

The relationship between voltage and time for the $\mathrm{GeP}_{5}$ electrode in the first lithiation/delithiation process is shown in Fig. S5. The onset potential of lithiation of $\mathrm{GeP}_{5}$ is known to be around $0.7 \mathrm{~V}$, which is the long plateau in the lithiation curve in Fig. S5 and corresponds to the conversion reaction between $\mathrm{Li}$ and $\mathrm{GeP}_{5}$ to form $\mathrm{Li}_{3} \mathrm{P}$, as shown in Eq. (1) ${ }^{9}$. The plateau at 0.18 $\mathrm{V}$ can be assigned to the alloy formation of $\mathrm{Li}_{4.4} \mathrm{Ge}$ as in Eq. (2) ${ }^{9}$. The selected particles were continuously focused, as shown in Fig. S6, where the letters for the images correspond to the letters marking points on the lithiation/de-lithiation curve in Fig. S5. Before the first lithiation, two particles were observed within the monitored area. The larger particle, marked by the yellow dashed line, became unclear from point $\mathrm{d}$, while the smaller particle, marked by the red dashed line, can hardly be observed in i-j. The reaction mechanism is shown below ${ }^{9}$ :

$$
15 \mathrm{Li}^{+}+15 \mathrm{e}^{-}+\mathrm{GeP}_{5} \rightarrow \mathrm{Ge}+5 \mathrm{Li}_{3} \mathrm{P}
$$




$$
\begin{gathered}
4.4 \mathrm{Li}^{+}+4.4 \mathrm{e}^{-}+\mathrm{Ge} \rightarrow \mathrm{Li}_{4.4} \mathrm{Ge} \\
\text { In total: } 19.4 \mathrm{Li}^{+}+19.4 \mathrm{e}^{-}+\mathrm{GeP}_{5} \rightarrow \mathrm{Li}_{4.4} \mathrm{Ge}+5 \mathrm{Li}_{3} \mathrm{P}
\end{gathered}
$$

The two plateaus in the de-lithiation curve in Fig. S5 can be assigned to the extraction of lithium from $\mathrm{Li}_{4}{ }_{4} \mathrm{Ge}$ and $\mathrm{Li}_{3} \mathrm{P}$. These reactions also can be found in Fig. S6, corresponding to the formation of the particle outlined in blue. 

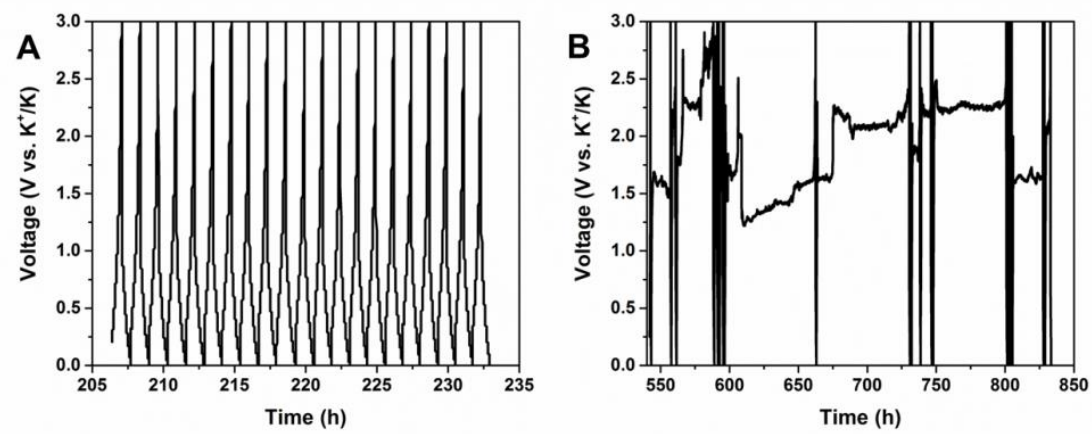

Fig. S7. Time-voltage profiles of the electrode in $\mathrm{KPF}_{6}$ electrolyte between a) 50-70 and b) 250270 cycles within the voltage range of 0.01-3 V. 


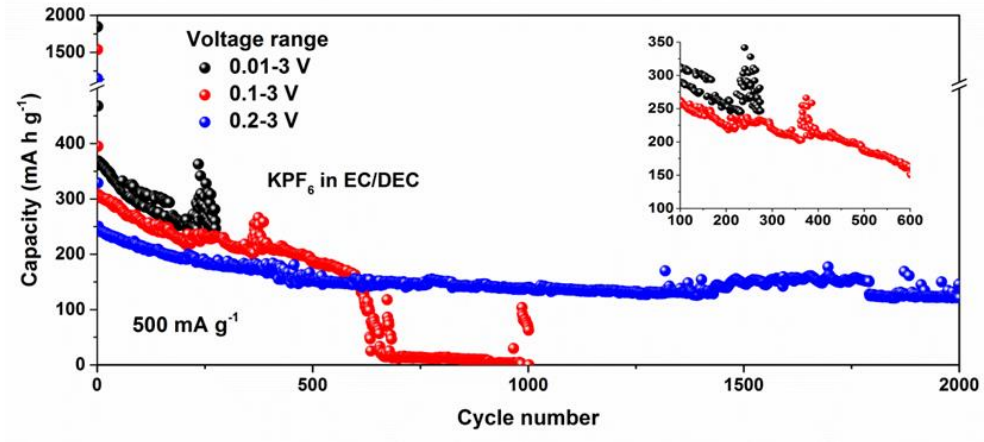

Fig. S8. Long-term cycling tests of the cells in various voltage ranges at $500 \mathrm{~mA} \mathrm{~g}^{-1}$ in $\mathrm{KPF}_{6}$ electrolyte, with the inset showing an enlargement of the indicated range. 


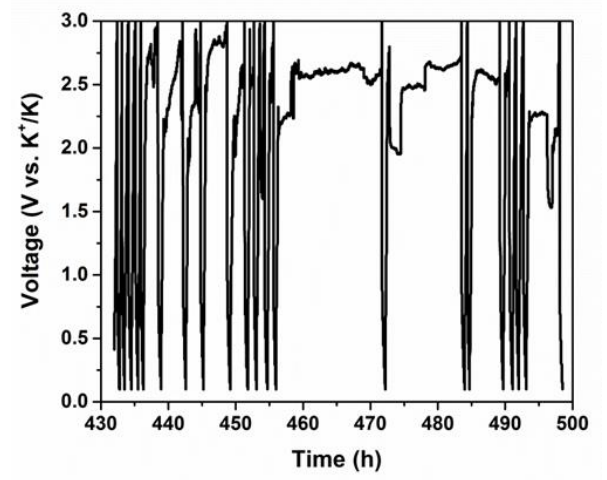

Fig. S9. Time-voltage profiles of the cells in $\mathrm{KPF}_{6}$ electrolyte between $360-380$ cycles within the voltage range of $0.1-3 \mathrm{~V}$. 


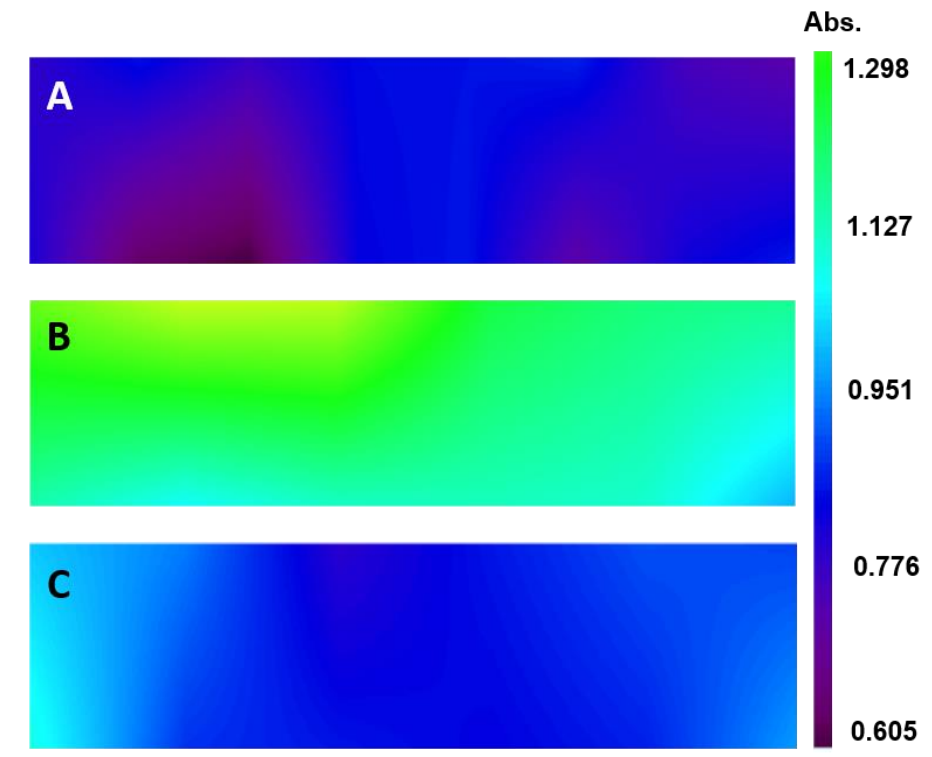

Fig. S10. FTIR mapping images of the selected areas on the surfaces of the electrodes: (A) pristine electrode, $(\mathrm{B}) \mathrm{KPF}_{6}$ with $\mathrm{EC} / \mathrm{DEC}$ electrolyte, and (C) KFSI with EC/DEC electrolyte. (Absorbance (Abs.) ranges were limited to the range from 0.6 to 1.3, with the colour scale based on the corrected absorbance adjusted to the same range.) 
Abs.

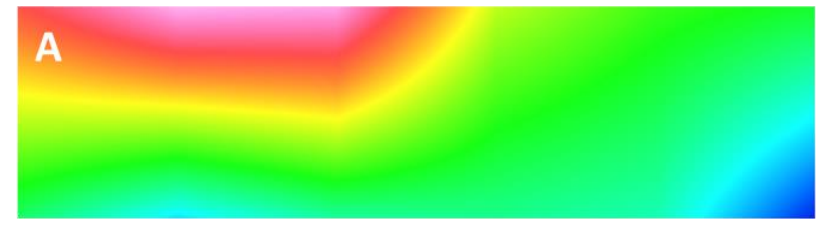

1.498

1.389

1.283

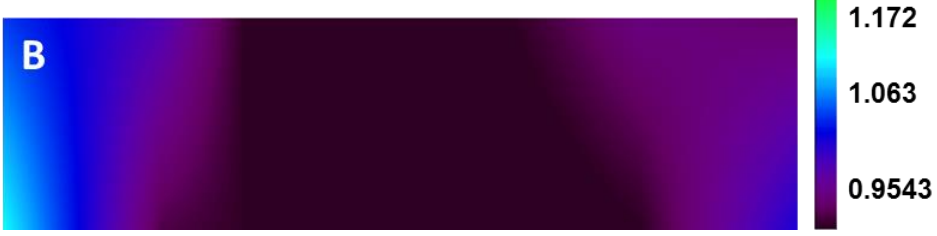

Fig. S11. FTIR mapping images of the selected areas on the surfaces of the electrodes: (A) $\mathrm{KPF}_{6}$ with EC/DEC electrolyte, and (B) KFSI with EC/DEC electrolyte. (Absorbance (Abs.) ranges were limited to the range from 0.9 to 1.5 , with the colour scale based on the corrected absorbance adjusted to the same range.) 


\section{Supplemental References}

1 W. K. Pang, S. Kalluri, V. K. Peterson, N. Sharma, J. Kimpton, B. Johannessen, H. K. Liu, S. X. Dou, Z. Guo. Interplay between Electrochemistry and Phase Evolution of the P2-type $\mathrm{Na}_{\mathrm{x}}\left(\mathrm{Fe}_{1 / 2} \mathrm{Mn}_{1 / 2}\right) \mathrm{O}_{2}$ Cathode for Use in Sodium-lon Batteries. Chem. Mater. 27, 3150-3158 (2015).

2 P. Hohenberg, W. Kohn. Inhomogeneous electron gas. Phys. Rev. 136, B864 (1964).

3 J. P. Perdew, K. Burke, M. Ernzerhof. Generalized gradient approximation made simple. Phys. Rev. Lett. 77, 3865 (1996).

4 B. Delley. Analytic energy derivatives in the numerical local - density - functional approach. J. Chem. Phys. 94, 72457250 (1991).

5 H. J. Monkhorst, J. D. Pack. Special points for Brillouin-zone integrations. Phys. Rev. B 13, 5188 (1976).

$6 \quad$ K. Xu, A. von Cresce, U. Lee. Differentiating contributions to "ion transfer" barrier from interphasial resistance and $\mathrm{Li}^{+}$desolvation at electrolyte/graphite interface. Langmuir 26, 11538-11543 (2010).

7 K. Xu, A. von Cresce. Interfacing electrolytes with electrodes in Li ion batteries. J. Mater. Chem. 21, 9849-9864 (2011).

8 T. A. Pham, K. E. Kweon, A. Samanta, V. Lordi, J. E. Pask. Solvation and Dynamics of Sodium and Potassium in Ethylene Carbonate from Ab Initio Molecular Dynamics Simulations. The Journal of Physical Chemistry C 121, 2191321920 (2017).

9 W. Li, H. Li, Z. Lu, L. Gan, L. Ke, T. Zhai, H. Zhou. Layered phosphorus-like GeP5: A promising anode candidate with high initial coulombic efficiency and large capacity for lithium ion batteries. Energy Environ. Sci. 8, 3629-3636 (2015). 